CERN-TH/98-132

hep-ph/9804349

\title{
Testing factorization of charmonium production
}

\author{
Gerhard A. Schuler ${ }^{a}$ \\ Theory Division, CERN, \\ CH-1211 Geneva 23, Switzerland \\ E-mail: Gerhard.Schuler@cern.ch
}

\begin{abstract}
Within the NRQCD factorization approach the production of heavy quarkonia can be calculated in perturbative QCD in terms of a few production-process-independent NRQCD matrix elements. We test the universality of these long-distance matrix elements by comparing their values as determined from charmonium production at $\mathrm{p} \overline{\mathrm{p}}$, ep, and $\mathrm{e}^{+} \mathrm{e}^{-}$colliders, and in $\mathrm{Z}^{0}, \Upsilon$, and $\mathrm{B}$ decays.
\end{abstract}

${ }^{a}$ Heisenberg Fellow.

CERN-TH/98-132

April 1998 


\section{Introduction}

The production of heavy quarkonia in high-energy reactions can be calculated in perturbative QCD in terms of a few non-perturbative parameters that are independent of the production process. More precisely, a factorization theorem holds, which expresses the cross section to produce a quarkonium $H$ as a series of terms

$$
\sigma(H)=\sum_{n} \sigma(Q \bar{Q}[n])\left\langle\mathcal{O}^{H}[n]\right\rangle,
$$

where $\sigma(Q \bar{Q}[n])$ is the cross section to produce a quark-antiquark pair with small relative momentum at distances $1 / m$ or smaller ( $m$ is the heavy-quark mass). The quantity $\left\langle\mathcal{O}^{H}[n]\right\rangle$ gives the probability for the heavy-quark pair of state $n$ to bind into the meson $H$. On the one hand, the binding occurs at distances much larger than $1 / m$, ensuring the existence of the factorized form (1D). On the other hand, the binding energy for neither charmonia nor bottomonia is large compared with the dynamical low-energy QCD scale $\Lambda_{\mathrm{QCD}}$. Hence the long-distance matrix elements (MEs) $\left\langle\mathcal{O}^{H}[n]\right\rangle$ cannot be computed perturbatively. However, there exists a power-counting scheme that provides a hierarchy of the MEs so that to any desired accuracy only a given number of terms contribute in (11).

The factorization (11) has been derived perturbatively using the so-called nonrelativistic QCD (NRQCD), an effective field theory appropriate for mesons containing a heavy-quark pair [1]. It therefore holds to leading order in $\Lambda_{\mathrm{QCD}} / \mathrm{m}$. Higher-twist corrections occur both in the production of the heavy-quark pairs and in their subsequent fragmentation into the meson. The importance of the NRQCD MEs $\left\langle\mathcal{O}^{H}[n]\right\rangle$ is governed by their scaling with $v=|\vec{v}|$, the relative velocity between the heavy quark $Q$ and the heavy antiquark $\bar{Q}$ within the bound state. Equation 11 is hence a double expansion in $v^{2}$ and $\alpha_{\mathrm{s}}\left(=\alpha_{\mathrm{s}}(\mu)\right.$ with $\left.\mu \sim m\right)$. The occurrence of $v^{2}$ as an expansion parameter is in line with the success of non-relativistic potential models in describing quarkonium spectroscopy and distinguishes quarkonia from heavy-light systems where $\Lambda_{\mathrm{QCD}} / m$ is the expansion parameter.

In the non-relativistic limit the quarkonium is a pure $Q \bar{Q}$ state with quantum numbers that match those of the meson, for example a $\mathrm{c}_{1}\left({ }^{3} S_{1}\right)$ state in the case of the $\mathrm{J} / \psi\left(J^{P C}=1^{--}\right)$and a $\mathrm{c}_{\mathrm{c}_{1}}\left({ }^{3} P_{J}\right)$ state in the case of the $J^{++} \chi_{\mathrm{c} J}$ mesons. The phenomenologically most important consequences of the NRQCD factorization approach (FA) are two-fold. First, two MEs contribute to $\chi_{\mathrm{c} J}$ production at leading order in $v^{2}$, namely $\left\langle\mathcal{O}_{1}^{\chi_{c J} J}\left({ }^{3} P_{J}\right)\right\rangle$ and $\left\langle\mathcal{O}_{8}^{\chi_{c J} J}\left({ }^{3} S_{1}\right)\right\rangle$. That is to say, besides the contribution from the leading Fock state, the colour-singlet $c \overline{\mathrm{c}}\left({ }^{3} P_{J}\right)$ state, there is, at leading order in $v^{2}$, a contribution where the heavy-quark pair is produced in a colour-octet state.

The second, perhaps more dramatic, consequence concerns the $1^{--}$mesons $\left(\mathrm{J} / \psi, \psi^{\prime}, \Upsilon(n S)\right)$ : although there is just one contribution corresponding to the short-distance production of the leading $Q \bar{Q}_{1}\left({ }^{3} S_{1}\right)$ Fock state for $v^{2} \rightarrow 0$, rel- 
ativistic corrections are sizeable, even partly dominating the production cross section, at least in the charm system: $v^{2} \sim 0.3$ is not small enough for gluon radiation from an almost point-like $Q \bar{Q}$ pair produced in the hard interaction (governed by $\alpha_{\mathrm{s}}$ ) to dominate gluon radiation from the pair at later times, when it has already expanded to the quarkonium size $\sim 1 /(m v)$ (governed by $v^{2}$ ); compare, for example, (3) and (5) below. Therefore contributions to the production of $1^{--}$mesons are important where the heavy-quark pair is produced at short distances in a colour-octet state. Colour-octet MEs first appear at relative order $v^{4}$. Different quarkonium-production reactions differ in the relative weights in which these MEs enter the $1^{--}$cross sections, i.e. by their short-distance parts $\sigma(Q \bar{Q}[n])$ with $n={ }^{3} S_{1}^{(1)},{ }^{3} S_{1}^{(8)},{ }^{1} S_{0}^{(8)}$, and ${ }^{3} P_{J}^{(8)}$ up to and including $O\left(v^{4}\right)$.

\section{Charmonium production at the Tevatron}

In $\mathrm{p} \overline{\mathrm{p}}$ collisions at high energies, charmonium particles come from the decay of b-flavoured hadrons and prompt production, the latter consisting of direct production and feed-down from higher charmonium states (except for $\psi^{\prime}$, the highest-lying charmonium state). Short-distance processes that produce direct $\psi(n S)$ 's in the leading colour-singlet state are

$$
\begin{aligned}
& \mathrm{g}+\mathrm{g} \rightarrow \mathrm{cc}_{1}\left({ }^{3} S_{1}\right)+\mathrm{g}: \quad \alpha_{\mathrm{s}}^{3} \frac{1}{p_{\perp}^{8}}, \\
& \mathrm{~g}+\mathrm{g} \rightarrow \mathrm{g}+\mathrm{g}^{\star}, \quad \mathrm{g}^{\star} \rightarrow \mathrm{c} \overline{\mathrm{c}}_{1}\left({ }^{3} S_{1}\right)+\mathrm{g}+\mathrm{g}: \quad \alpha_{\mathrm{s}}^{5} \frac{1}{p_{\perp}^{4}}
\end{aligned}
$$

where the power of $\alpha_{\mathrm{s}}$ and the $p_{\perp}$ fall-off (at $90^{\circ}$ in the partonic c.m.s.) have been indicated. At large $p_{\perp}$, (3) dominates and can be calculated by folding the $\mathrm{gg} \rightarrow \mathrm{g} X$ subprocess with the $\mathrm{g} \rightarrow \psi X$ fragmentation function [2]. With values of the colour-singlet MEs as obtained from potential model calculations, (2) and (3) underestimate the observed yield of direct $\mathrm{J} / \psi$ and $\psi^{\prime}$ mesons by a factor $\sim$ 30. The data can, however, be accounted for (Fig. 1) if the short-distance production of colour-octet $Q \bar{Q}$ states is included [3, 4]

$$
\begin{aligned}
& \mathrm{g}+\mathrm{g} \rightarrow \mathrm{c} \overline{\mathrm{c}}_{8}[n]+\mathrm{g} \quad n={ }^{1} S_{0},{ }^{3} P_{J}: \quad \alpha_{\mathrm{s}}^{3} v^{4} \frac{4 m^{2}}{p_{\perp}^{6}} \\
& \mathrm{~g}+\mathrm{g} \rightarrow \mathrm{g}+\mathrm{g}^{\star}, \quad \mathrm{g}^{\star} \rightarrow \mathrm{c}^{\star} \overline{\mathrm{c}}_{8}\left({ }^{3} S_{1}\right): \quad \alpha_{\mathrm{s}}^{3} v^{4} \frac{1}{p_{\perp}^{4}}
\end{aligned}
$$

The $p_{\perp}$ shapes of these two mechanisms are sufficiently different over the measured range to isolate the ${ }^{3} S_{1}$ colour-octet ME, but the remaining two MEs can be extracted only in the linear combination

$$
M_{a}^{H}=\left\langle\mathcal{O}_{8}^{H}\left({ }^{1} S_{0}\right)\right\rangle+\frac{a}{m_{\mathrm{c}}^{2}}\left\langle\mathcal{O}_{8}^{H}\left({ }^{3} P_{0}\right)\right\rangle
$$




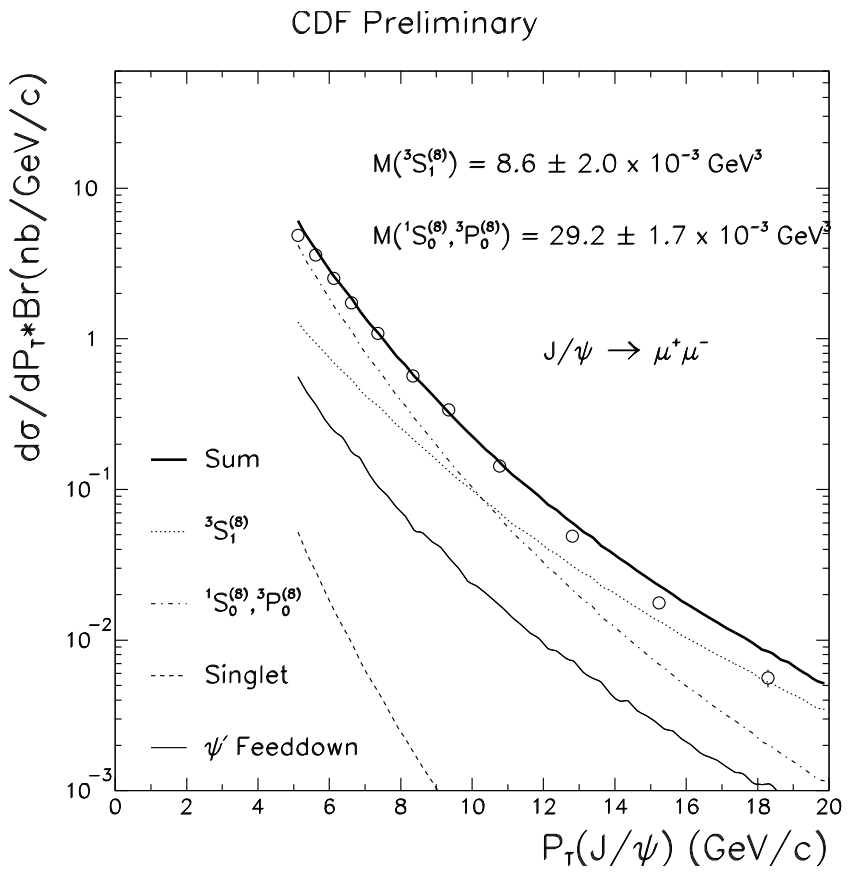

Figure 1: Fits to direct $\mathrm{J} / \psi$ production at the Tevatron [5].

where $a \approx 3.5$. The numerical values are indeed consistent with the expectation from velocity scaling, see table 11. The values are, however, subjected to a number of uncertainties:

- The value of the charm-quark mass; it affects mainly the overall normalization and should always be supplied when quoting values for the ME.

- The parametrization of the parton-distribution functions (PDF) and the choice of the factorization scale; both influence the normalization as well as the shape of the $p_{\perp}$ distribution.

- The value of $\Lambda_{\mathrm{QCD}}$ (which is not independent of the PDF) and the renormalization scale $\mu_{R}$; note that processes (피 and (5) start at high order in $\alpha_{\mathrm{s}}$. The values of $\Lambda_{\mathrm{QCD}}$ and $\mu_{R}$ affect also the $p_{\perp}$ shape, since $\mu_{R}^{2} \sim m^{2}+p_{\perp}^{2}$.

- The lack of higher-order perturbative QCD corrections. Particularly important are: (i) initial-state radiation and intrinsic $k_{\perp}$ effects since they may modify the slopes of (田) and (5) considerably [6]; (ii) Colour-singlet production via the $O\left(\alpha_{\mathrm{s}}^{4}\right)$ reaction $\mathrm{g}+\mathrm{g} \rightarrow \mathrm{c}_{1}\left({ }^{3} S_{1}\right)+\mathrm{g}+\mathrm{g}$, since it has the same $p_{\perp}$ fall-off as (画) [7].

- Higher-order $v^{2}$ corrections are important close to boundaries of partonic thresholds $[8]$. 


\begin{tabular}{|c|c|c|c|c|}
\cline { 2 - 5 } \multicolumn{1}{c|}{} & $\left\langle\mathcal{O}_{1}^{H}\left({ }^{3} S_{1}\right)\right\rangle$ & $\left\langle\mathcal{O}_{1}^{H}\left({ }^{3} P_{1}\right)\right\rangle / m_{\mathrm{c}}^{2}$ & $\left\langle\mathcal{O}_{8}^{H}\left({ }^{3} S_{1}\right)\right\rangle$ & $\mathcal{M}_{3.5}^{H}$ \\
\hline$\psi(n S)$ & 1 & $v^{6}$ & $v^{4}$ & $v^{4}$ \\
\hline $\mathrm{J} / \psi$ & 1.16 & - & $1.1 \times 10^{-2}$ & $4.4 \times 10^{-2}$ \\
\hline$\psi^{\prime}$ & 0.76 & - & $3.8 \times 10^{-3}$ & $1.8 \times 10^{-2}$ \\
\hline \hline$\chi_{\mathrm{c} J}$ & $v^{6}$ & $v^{2}$ & $v^{2}$ & $v^{4}$ \\
\hline$\chi_{\mathrm{c} 1}$ & - & $0.32 / 1.48^{2}$ & $2.4 \times 10^{-2}$ & - \\
\hline
\end{tabular}

Table 1: Scaling of NRQCD matrix elements with $v$ (modulo $v^{3}$ ) and numerical values in $\mathrm{GeV}^{-3}$ as obtained from a potential-model calculation (second and third row) [9] and fits to the Tevatron $p_{\perp}$ distribution (last two rows) [5].

- Higher-twist corrections. At large $p_{\perp}$ these are probably small for the shortdistance cross sections. However, sizeable corrections may be present for the fragmentation functions at the input scale.

- Values of the colour-singlet matrix elements; at the Tevatron this is important for $\chi_{\mathrm{c} J}$ production only, i.e. the extracted value of $\left\langle\mathcal{O}_{8}^{\chi_{c} J}\left({ }^{3} S_{1}\right)\right\rangle$.

From the above discussion we expect the current determination (table 1) of $\left\langle\mathcal{O}_{8}^{\psi(n S)}\left({ }^{3} S_{1}\right)\right\rangle$ to be on the low side, $M_{3.5}^{\psi(n S)}$ to be overestimated, and a strong correlation between $\left\langle\mathcal{O}_{8}^{\chi_{c 1}}\left({ }^{3} S_{1}\right)\right\rangle$ and $\left\langle\mathcal{O}_{1}^{\chi_{c 1}}\left({ }^{3} P_{1}\right)\right\rangle$.

Although it is reassuring that the FA can accommodate the data, the result is not too surprising given that we have two additional mechanisms that scale as $1 / p_{\perp}^{4}$ and $1 / p_{\perp}^{6}$, respectively, with free normalizations. Additional consistency checks are therefore needed. The polarization of the $\mathrm{J} / \psi$, measurable via its decay into $\mu^{+} \mu^{-}$, is one such test since it is uniquely predicted without the need of additional long-distance MEs up to $O\left(v^{4}\right)$ [4]. Polarization measurements should soon become available at the Tevatron. Here we focus on another aspect of the FA, namely the universality of the ME. To this end we compare MEs extracted from various reactions.

\section{Z-decay}

The Feynman diagrams for quarkonium production in $\mathrm{e}^{+} \mathrm{e}^{-}$annihilation are shown in Fig. 2. Results of a recent prediction [10] are compared with LEP data [11, [12] in table 2. At $\mathrm{e}^{+} \mathrm{e}^{-}$c.m. energies $\sqrt{s}$ that are large with respect to the heavy-quark mass, for example in $\mathrm{Z}^{0}$ decays, contributions with additional gluons (lower diagrams in Fig. 2) are suppressed by powers of $m / \sqrt{s}$. The 

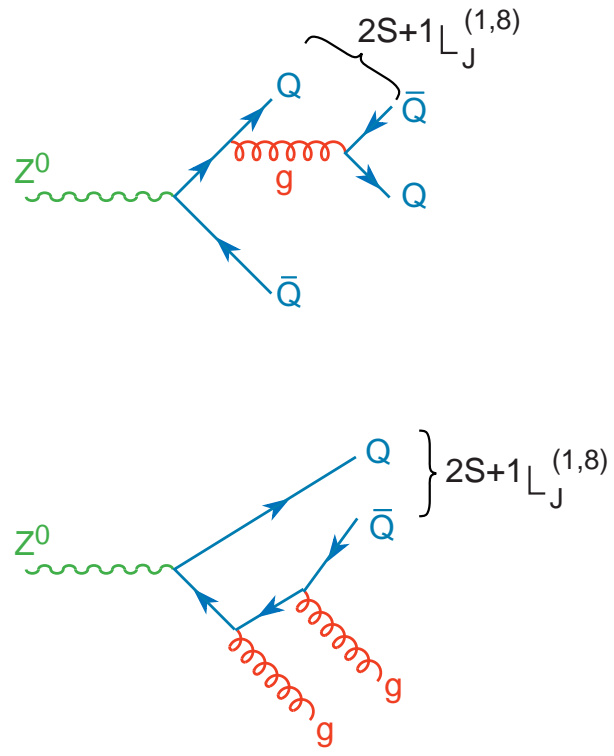
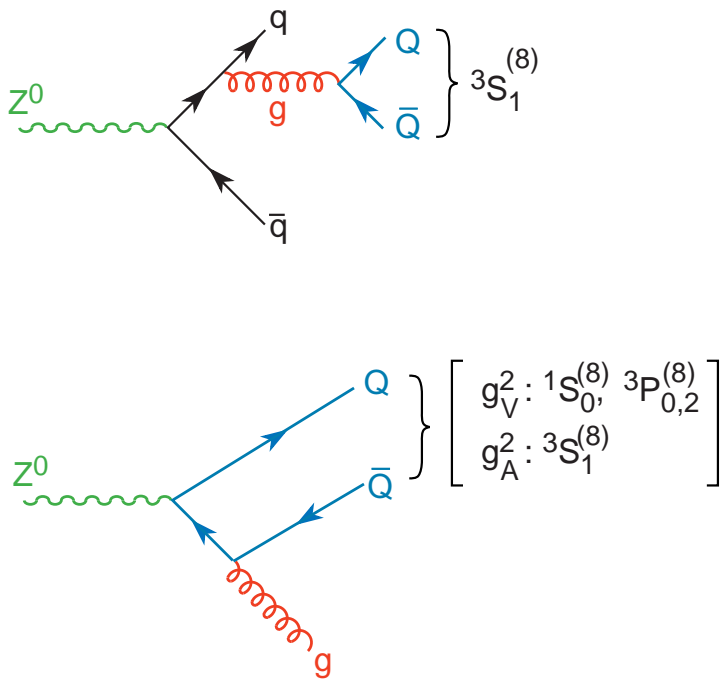

Figure 2: Feynman diagrams for quarkonium production in $\mathrm{Z}^{0}$ decays.

dominant contributions arise from $Q \bar{Q}$ production in the leading (colour-singlet) Fock state (upper left diagram in Fig. 2) and the production of a $Q \bar{Q}_{8}\left({ }^{3} S_{1}\right)$ pair through gluon splitting (upper right diagram in Fig. 2). The former contribution is the only one present in the so-called colour-singlet model (CSM). As table 2 reveals, this colour-singlet contribution alone seems low with respect to the $\mathrm{J} / \psi$ data, although still compatible, within errors.

The data are well described, after inclusion of the colour-octet part, with a value for $\left\langle\mathcal{O}_{8}^{\mathrm{J} / \psi}\left({ }^{3} S_{1}\right)\right\rangle$ as extracted from fits to the Tevatron $p_{\perp}$ distribution. In the case of $\Upsilon$ production, the statistics per experiment is too low to allow for definite conclusions. Further improvement can be expected because not all $\mathrm{Z}^{0}$ data have yet been analysed. Since the error of the data is statistics-dominated, a combined LEP analysis would be welcome. Information on colour-octet MEs other than $\left\langle\mathcal{O}_{8}^{\mathrm{J} / \psi}\left({ }^{3} S_{1}\right)\right\rangle$ will come only from $\mathrm{e}^{+} \mathrm{e}^{-}$annihilation at lower energies, BES, CESR, and B-factories.

The LEP data are also consistent with the expectation of the colourevaporation model (CEM), a model of quarkonium formation that assigns precisely one process-independent, long-distance factor $f^{H}=\left\langle\mathcal{O}^{H}[n]\right\rangle$ to each quarkonium particle $H$ [13]. The cross section $\sigma(Q \bar{Q}[n])$, see (11), is obtained by integrating the $Q \bar{Q}$ production cross section up to the threshold of the production of a pair of heavy-light mesons. With parameters $f^{H}$ extracted from fixed-target hadroproduction, the CEM is also able to describe the Tevatron $p_{\perp}$ distributions [14]. 


\begin{tabular}{|c|ccc|cccc|}
\cline { 2 - 8 } \multicolumn{1}{c|}{} & CSM & CEM & NRQCD & DELPHI & OPAL & ALEPH & L3 \\
\hline $\mathrm{J} / \psi$ & 7.8 & 23 & 26 & $44_{-30}^{+36}$ & $19 \pm 10$ & $30 \pm 9$ & $27 \pm 12$ \\
\hline$\sum \Upsilon$ & 1.7 & 1.7 & 6.6 & $<124$ & $10 \pm 5$ & $<7.3$ & $<7.6$ \\
\hline
\end{tabular}

Table 2: Branching ratios and 95\% CL upper limits in $10^{-5}$ of $Z^{0}$ decays: production of the sum of the three lowest-lying $\Upsilon$ states and prompt $\mathrm{J} / \psi$ production (i.e. excluding B decays but including feed-down from $\chi_{\mathrm{c} J}$ and $\psi^{\prime}$ ).

\section{Low-energy electron-positron annihilation}

In low-energy $\mathrm{e}^{+} \mathrm{e}^{-}$annihilation, $\mathrm{J} / \psi$ production is unique in the sense that it is the only process known so far where the colour-octet ${ }^{1} S_{0}$ and ${ }^{3} P_{0}$ MEs enter with widely different weights. At low energies, the dominant contributions involve a cc pair plus one or two perturbative gluons (lower Feynman diagrams in Fig. 2) [15, 16]. The cross sections are readily evaluated and results presented in table 3 and Fig. 3 .

Observe first that the ME combination (6) enters with $a$ varying between 11.3 and 3.8 in $5<\sqrt{s}<10.6 \mathrm{GeV}$. The central values of the experimental data yield for $\alpha_{\mathrm{s}}=0.278, m_{\mathrm{c}}=1.5 \mathrm{GeV}$, and $\left\langle\mathcal{O}_{1}^{\mathrm{J} / \psi}\left({ }^{3} S_{1}\right)\right\rangle=1.16 \mathrm{GeV}^{3}$

$$
\left\langle\mathcal{O}_{8}^{\mathrm{J} / \psi}\left({ }^{1} S_{0}\right)\right\rangle=4.0 \times 10^{-4} \mathrm{GeV}^{3}, \quad \frac{\left\langle\mathcal{O}_{8}^{\mathrm{J} / \psi}\left({ }^{3} P_{0}\right)\right\rangle}{m_{\mathrm{c}}^{2}}=9.3 \times 10^{-3} \mathrm{GeV}^{3},
$$

and hence $\mathcal{M}_{3.5}^{\mathrm{J} / \psi}=3.3 \times 10^{-2} \mathrm{GeV}^{3}$ (unprimed curves in Fig. 3). The last number is somewhat smaller than the naive Tevatron estimate, see table 1 .

Our numbers are similar to those found in [16]. There it was claimed that $\left\langle\mathcal{O}_{8}^{\mathrm{J} / \psi}\left({ }^{3} P_{0}\right)\right\rangle / m_{\mathrm{c}}^{2}$ can be extracted very precisely from low-energy $\mathrm{e}^{+} \mathrm{e}^{-}$data while $\left\langle\mathcal{O}_{8}^{\mathrm{J} / \psi}\left({ }^{1} S_{0}\right)\right\rangle$ is hardly constrained, based on the dominance of $R_{8}\left({ }^{3} P_{0}\right)$ over $R_{1}$ and $R_{8}\left({ }^{1} S_{0}\right)$ at low energies and the fact that $R_{8}\left({ }^{1} S_{0}\right)$ is a small contribution for all $\sqrt{s}$. Indeed, varying $\alpha_{\mathrm{s}}$ in $(0.24,0.30), m_{\mathrm{c}}$ in $(1.4,1.6) \mathrm{GeV}$, and $\left\langle\mathcal{O}_{1}^{\mathrm{J} / \psi}\left({ }^{3} S_{1}\right)\right\rangle$ in $(0.80,1.52) \mathrm{GeV}^{3}$ yields, for the central experimental values, the following ranges:

$$
-1.2<\frac{10^{2}}{\mathrm{GeV}^{3}}\left\langle\mathcal{O}_{8}^{\mathrm{J} / \psi}\left({ }^{1} S_{0}\right)\right\rangle<1.2, \quad 1.1>\frac{10^{2}}{\mathrm{GeV}^{3} m_{\mathrm{c}}^{2}}\left\langle\mathcal{O}_{8}^{\mathrm{J} / \psi}\left({ }^{3} P_{0}\right)\right\rangle>0.78,
$$

implying $2.6<10^{2} \mathcal{M}_{3.5}^{\mathrm{J} / \psi} / \mathrm{GeV}^{3}<4.5$.

\footnotetext{
${ }^{1}$ These are the maximal ranges obtained by varying one parameter at a time; the uncertainty associated with each of the three parameters is about the same.
} 


\begin{tabular}{|c|c|c|c|c|}
\hline$\sqrt{s}$ & $R[$ exp. $]$ & $R_{1}$ & $R_{8}\left({ }^{1} S_{0}\right)$ & $R_{8}\left({ }^{3} P_{0}\right)$ \\
\hline \multirow{2}{*}{$5 \mathrm{GeV}$} & \multirow{2}{*}{$60 \pm 40$} & $3.3\left\langle\mathcal{O}_{1}^{\mathrm{J} / \psi}\left({ }^{3} S_{1}\right)\right\rangle$ & $530\left\langle\mathcal{O}_{8}^{\mathrm{J} / \psi}\left({ }^{1} S_{0}\right)\right\rangle$ & $\frac{6000}{m_{c}^{2}}\left\langle\mathcal{O}_{8}^{\mathrm{J} / \psi}\left({ }^{3} P_{0}\right)\right\rangle$ \\
\cline { 3 - 5 } & & 3.8 & 0.2 & 56 \\
\cline { 2 - 5 } & 20 & 3.8 & 39 & -23 \\
\hline \hline \multirow{2}{*}{$10.6 \mathrm{GeV}$} & \multirow{2}{*}{$10 \pm 4$} & $3.4\left\langle\mathcal{O}_{1}^{\mathrm{J} / \psi}\left({ }^{3} S_{1}\right)\right\rangle$ & $170\left\langle\mathcal{O}_{8}^{\mathrm{J} / \psi}\left({ }^{1} S_{0}\right)\right\rangle$ & $\frac{640}{m_{c}^{2}}\left\langle\mathcal{O}_{8}^{\mathrm{J} / \psi}\left({ }^{3} P_{0}\right)\right\rangle$ \\
\cline { 3 - 5 } & & 3.9 & 0.07 & 6.0 \\
\cline { 2 - 5 } & 14 & 3.9 & 12.5 & -2.5 \\
\hline
\end{tabular}

Table 3: Cross section of prompt $\mathrm{J} / \psi$ production in $\mathrm{e}^{+} \mathrm{e}^{-}$annihilation (excluding $\mathrm{b}$ decays) in units of $10^{4} \sigma_{\mu \mu}$ : Data [17] $\left(R\left[\right.\right.$ exp.]), colour-singlet contribution $\left(R_{1}\right)$, and colour-octet contributions through ${ }^{1} S_{0}$ and ${ }^{3} P_{J}$ intermediate $c \bar{c}$ pairs. The PLUTO data $\sigma=(31 \pm 21)$ pb have been multiplied by $(1-0.23) 6 / 7$ to remove the $\psi^{\prime}$ contribution and to update the leptonic branching ratio $\mathrm{J} / \psi \rightarrow \mu^{+} \mu^{-}$. The theoretical values are for $\alpha_{\mathrm{s}}=0.278, m_{\mathrm{c}}=1.5 \mathrm{GeV}$, the colour-singlet ME as given in table 1 and the colour-octet MEs (7). Also given are the results of one of the extreme solutions (9).

However, a few remarks are in order. First, the large experimental errors forbid definite conclusions 2 . Even for fixed theoretical parameters $\alpha_{\mathrm{s}}, m_{\mathrm{c}}$, $\left\langle\mathcal{O}_{1}^{\mathrm{J} / \psi}\left({ }^{3} S_{1}\right)\right\rangle$ only the following, much wider range is obtained:

$$
-0.73<\frac{10^{2}}{\mathrm{GeV}^{3}}\left\langle\mathcal{O}_{8}^{\mathrm{J} / \psi}\left({ }^{1} S_{0}\right)\right\rangle<7.4, \quad 2.2>\frac{10^{2}}{\mathrm{GeV}^{3} m_{\mathrm{c}}^{2}}\left\langle\mathcal{O}_{8}^{\mathrm{J} / \psi}\left({ }^{3} P_{0}\right)\right\rangle>-0.38,
$$

implying

$$
\mathcal{M}_{3.5}^{\mathrm{J} / \psi}=(3.3 \pm 2.7) \times 10^{-2} \mathrm{GeV}^{3} .
$$

Insisting in $\left\langle\mathcal{O}_{8}^{\left.\mathrm{J} / \psi^{1} S_{0}\right)}\right\rangle>0$, the minimally allowed value of $\mathcal{M}_{3.5}^{\mathrm{J} / \psi}$ increases from $0.57 \times 10^{-2} \mathrm{GeV}^{3}$ to $1.1 \times 10^{-2} \mathrm{GeV}^{3}$. One pair of MEs fulfilling (9) $\left(\left\langle\mathcal{O}_{8}^{\mathrm{J} / \psi\left({ }^{1} S_{0}\right)}\right\rangle=\right.$ $\left.0.05 \mathrm{GeV}^{3},\left\langle\mathcal{O}_{8}^{\mathrm{J} / \psi}\left({ }^{3} P_{0}\right)\right\rangle / m_{\mathrm{c}}^{2}=9.5 / 10^{5} \mathrm{GeV}^{3}\right)$ is shown in Fig. 圂 (primed curves). If we allow in addition the (independent) variation of the theoretical parameters, even negative values of $\mathcal{M}_{3.5}^{\mathrm{J} / \psi}$ are not excluded.

Negative values for $\left\langle\mathcal{O}_{8}^{\mathrm{j} / \psi}\left({ }^{3} P_{0}\right)\right\rangle$ are not per se excluded, although at first sight these would lead to negative cross sections at low c.m. energies since the cross section of $c \overline{\mathrm{C}}_{8}\left({ }^{3} P_{J}\right)$ production grows at low energies while all others vanish.

\footnotetext{
${ }^{2}$ Recall that PLUTO [17] observes an excess of only 4 prompt $\mathrm{e}^{+} \mathrm{e}^{-} \rightarrow \mathrm{J} / \psi X$ events; out of these, only the two events with both decay muons reconstructed have been used here.
} 


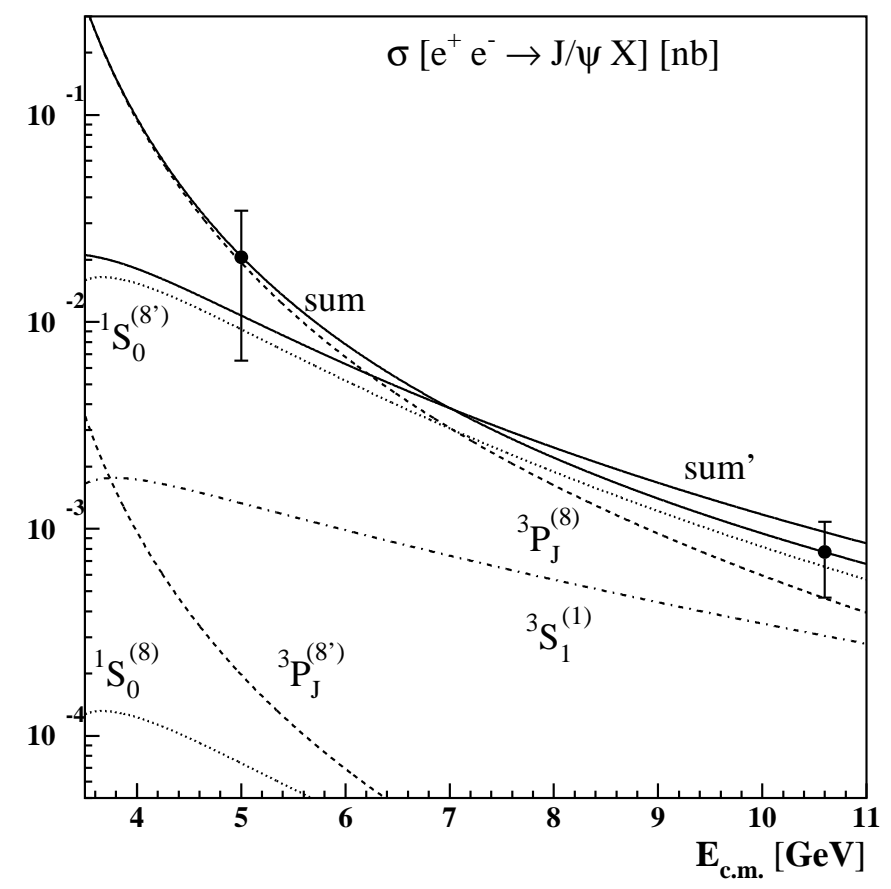

Figure 3: Fits to direct $\mathrm{J} / \psi$ production in $\mathrm{e}^{+} \mathrm{e}^{-}$annihilation.

However, the $\mathrm{e}^{+} \mathrm{e}^{-} \rightarrow \mathrm{J} / \psi X$ cross section at low energies is no longer given by the fixed-order calculation. The reason is the singular behaviour of the ${ }^{3} P_{J}$ cross sections: for $\xi=2 m_{\mathrm{c}} / \sqrt{s} \rightarrow 1$, these diverge as $1 /(1-\xi)$. Clearly this indicates the breakdown of the fixed-order in $v^{2}$ calculation: $v^{4} /(1-\xi)$ is no longer a small parameter and higher-order terms in $v^{2}$ grow more rapidly as $\xi \rightarrow 1$. The energy dependence at low energies can only be described once these terms of higher order in $v^{2}$ are resummed. Eventually, as $1-\xi<v^{2}$, higher-twist contributions become important, too. Of course, the result of the fixed-order calculation can still be used if we smear over a sufficiently wide region in $\sqrt{s}$.

Near threshold the cross section is given by

$$
\begin{aligned}
\sigma\left[\mathrm{e}^{+} \mathrm{e}^{-} \rightarrow \mathrm{J} / \psi+x\right]_{\text {direct }} \stackrel{\xi \rightarrow 1}{\longrightarrow} \\
\sigma_{0}\left\{\left\langle\mathcal{O}_{1}^{\mathrm{J} / \psi}\left({ }^{3} S_{1}\right)\right\rangle\left[\delta(1-\xi)+\left(\frac{\alpha_{\mathrm{s}}}{\pi}\right)^{2} \frac{32}{81}(1-\xi)\right]\right. \\
\left.+\frac{\alpha_{\mathrm{s}}}{\pi}\left[8(1-\xi)\left\langle\mathcal{O}_{8}^{\mathrm{J} / \psi}\left({ }^{1} S_{0}\right)\right\rangle+\frac{8}{3} \frac{1}{1-\xi} \frac{1}{m_{\mathrm{c}}^{2}} \sum_{J=0}^{2}\left\langle\mathcal{O}_{8}^{\mathrm{J} / \psi}\left({ }^{3} P_{J}\right)\right\rangle\right]\right\},
\end{aligned}
$$

where $\sigma_{0}=\pi^{3} \alpha_{\mathrm{em}}^{2} e_{c}^{2} /\left(6 m_{\mathrm{c}}^{5}\right), e_{c}=2 / 3$. The apparent singularity at $\xi=1$ can be absorbed through the factorization-scale dependence of the leading colour-singlet 
ME owing to the evolution equation derived in [1, 18

$$
\Lambda \frac{\mathrm{d}}{\mathrm{d} \Lambda} \mathcal{O}_{1}\left({ }^{3} S_{1}\right)=\frac{8 \alpha_{\mathrm{s}}}{3 \pi m_{\mathrm{c}}^{2}} \sum_{J=0}^{2} \mathcal{O}_{8}\left({ }^{3} P_{J}\right)-\frac{8 C_{F} \alpha_{\mathrm{s}}}{3 \pi m_{\mathrm{c}}^{2}} \mathcal{P}_{1}\left({ }^{3} S_{1}\right)
$$

Hence a finite cross section is arrived at when averaging the threshold region over a range of order $m_{\mathrm{c}}$.

\section{B decay}

The $\mathrm{B}$ meson is known to decay into charmonia with branching ratios between 0.1 and $1 \%$ [19. Branching ratios into charmonia of the other b-flavoured hadrons are not known. The hard process is given by

$$
\mathrm{b} \rightarrow \mathrm{c} \overline{\mathrm{c}}_{c}[n]+X_{s}, \quad n={ }^{1} S_{0},{ }^{3} S_{1},{ }^{3} P_{1}, D \text {-waves }, \ldots,
$$

where $X_{s}$ is a system containing a strange quark and, as usual, the subscript $c$ indicates a colour-singlet $(c=1)$ or colour-octet $(c=8)$ heavy-quark pair. Calculations of $\mathrm{B}$ decays into charmonium states based on (13) are valid to leading order in $v^{2}$ and leading order in $\Lambda_{\mathrm{QCD}} / m_{\mathrm{b}}$. Using the results of [20], we can find the $\mathrm{B}$ into charmonium decay-branching ratios in terms of the semileptonic branching ratio

$$
\begin{aligned}
\operatorname{Br}[\mathrm{B} \rightarrow H+X]= & \operatorname{Br}\left[\mathrm{b} \rightarrow \mathrm{c} \ell \nu_{\ell}\right] \frac{4 \pi^{2}}{9 m_{\mathrm{b}}^{3}} \frac{R\left(m_{\mathrm{c}} / m_{\mathrm{b}}\right)}{f\left(m_{\mathrm{c}} / m_{\mathrm{b}}\right)} \\
& \times\left\{\left[2 C_{+}-C_{-}\right]^{2} \hat{\Gamma}_{1}^{H}+\left[C_{+}+C_{-}\right]^{2} \frac{3}{2} \hat{\Gamma}_{8}^{H}\right\} .
\end{aligned}
$$

Here $f(r)$ is the phase-space function for the b semileptonic decay

$$
f(r)=1-8 r^{2}+8 r^{6}-r^{8}-24 r^{4} \ln (r),
$$

$R(z)$ is the phase-space function for $\mathrm{b} \rightarrow H+X$,

$$
R(z)=\frac{1}{z}\left[1-(2 z)^{2}\right]^{2}
$$

and

$$
\begin{aligned}
\hat{\Gamma}_{c}^{H}= & \left(1+8 z^{2}\right)\left\langle\mathcal{O}_{c}^{H}\left({ }^{3} S_{1}\right)\right\rangle-\frac{1}{3 m_{\mathrm{c}}^{2}}\left\langle\mathcal{P}_{c}^{H}\left({ }^{3} S_{1}\right)\right\rangle \\
& +3\left\langle\mathcal{O}_{c}^{H}\left({ }^{1} S_{0}\right)\right\rangle+\left(1+8 z^{2}\right) \frac{2}{m_{\mathrm{c}}^{2}}\left\langle\mathcal{O}_{c}^{H}\left({ }^{3} P_{1}\right)\right\rangle .
\end{aligned}
$$

Of course, for a particular charmonium particle $H$ and a specified colour state $c$ not all MEs in (17) contribute to any given order in $v^{2}$, see table 1. The second 
ME in (17), $\left\langle\mathcal{P}_{c}^{H}\left({ }^{3} S_{1}\right)\right\rangle$, is a $v^{2}$ correction to $\left\langle\mathcal{O}_{c}^{H}\left({ }^{3} S_{1}\right)\right\rangle$ and will be neglected in the following.

Finally, $C_{ \pm}$are factorization-scale-dependent Wilson coefficients describing the evolution from the $\mathrm{W}$-mass scale down to a scale of the order of the bquark mass. Note that the coefficient of the colour-octet contribution is strongly enhanced compared with the colour-singlet one:

$$
\frac{3}{2}\left(\frac{C_{+}+C_{-}}{2 C_{+}-C_{-}}\right)^{2} \approx 50 .
$$

In the following we take as central values $C_{+}+C_{-}=2.2,2 C_{+}-C_{-}=0.38$, $m_{\mathrm{c}}=1.48 \mathrm{GeV}, m_{\mathrm{b}}=5.0 \mathrm{GeV}, \mathrm{Br}\left[\mathrm{b} \rightarrow \mathrm{c} \ell \nu_{\ell}\right]=10.3 \%$. In contrast to the colour-octet Wilson coefficient, the colour-singlet one is strongly scale sensitive, since it is given as the difference of two large numbers. In order to bracket its uncertainty we shall vary $2 C_{+}-C_{-}$between $0.19=0.38 / 2$ and 0.645 . With the latter value, $\mathrm{Br}[\mathrm{B} \rightarrow \mathrm{J} / \psi X]$ is saturated with only the colour-singlet contribution (see below). Varying the colour-singlet coefficient in $(0.19,0.645)$ gives the range from 17 to 201 for the ratio (18).

As is true for most quarkonium production reactions, B decays into charmonia are sensitive to only the combination (6) of colour-octet ME. Moreover, the value of $a$ is similar to the one at the Tevatron, $a=2\left(1+8 z^{2}\right) \approx 3.4$. This follows immediately upon using the approximate symmetry relation $\left\langle\mathcal{O}_{8}^{H}\left({ }^{3} P_{1}\right)\right\rangle \approx 3\left\langle\mathcal{O}_{8}^{H}\left({ }^{3} P_{0}\right)\right\rangle$.

Restricting to the lowest order in $v^{2}$ terms, separately for the colour-singlet and colour-octet contributions, we find the following direct branching ratios

$$
\begin{array}{cccl}
\mathrm{Br}[\mathrm{B} \rightarrow \psi+X]= & 0.24 \frac{\left\langle\mathcal{O}_{1}^{\psi}\left({ }^{3} S_{1}\right)\right\rangle}{\mathrm{GeV}^{3}} & +12 \frac{\left\langle\mathcal{O}_{8}^{\psi}\left({ }^{3} S_{1}\right)\right\rangle}{\mathrm{GeV}^{3}} & +21 \frac{\mathcal{M}_{3.4}^{\psi}}{\mathrm{GeV}^{3}} \\
\mathrm{~J} / \psi: 0.80 \pm 0.08 & 0.28(0.07,0.80) & 0.13 & 0.92 \\
\psi^{\prime}: 0.34 \pm 0.05 & 0.18(0.04,0.52) & 0.046 & 0.38 \\
& 0.10 & 0.024 & 0.20 \quad(\kappa=0.53)
\end{array}
$$

and

$$
\begin{array}{cccc}
\mathrm{Br}\left[\mathrm{B} \rightarrow \chi_{\mathrm{c} J}+X\right]= & 0.48 \frac{\left\langle\mathcal{O}_{1}^{\chi_{\mathrm{c} 1}}\left({ }^{3} P_{1}\right)\right\rangle}{m_{\mathrm{c}}^{2} \mathrm{GeV}^{3}} \delta_{1, J} & +12 \frac{\left\langle\mathcal{O}_{8}^{\left.\chi_{\mathrm{c} J}\left({ }^{3} S_{1}\right)\right\rangle}\right.}{\mathrm{GeV}^{3}} & \\
\chi_{\mathrm{c} 1}: 0.37 \pm 0.07 & 0.070(0.017,0.20) & 0.28 & \\
\chi_{\mathrm{c} 2}: 0.23 \pm 0.10 & 0.046 & 0.19 & (\kappa=0.66) \\
& 0 & 0.47 & (\kappa=0.63)
\end{array}
$$

The experimental numbers (first rows) are the CLEO measurements [19 of the direct branching ratios (obtained by subtracting the feed-down from $\psi^{\prime} \rightarrow \chi_{\mathrm{c} J} X$ 
and $\left.\chi_{\mathrm{c} J} \rightarrow \mathrm{J} / \psi \gamma\right)$. The numbers in brackets denote the range of the coloursinglet contribution obtained by varying $2 C_{+}-C_{-}$in the range specified above. The numbers quoted for $\kappa \neq 1$ are obtained by using meson masses rather than quark masses in the phase-space function, i.e. by multiplying (14) by $\kappa=R\left(\left[m_{H} / 2\right] / m_{\mathrm{B}}\right) / R\left(m_{\mathrm{c}} / m_{\mathrm{b}}\right)$. Clearly there are other $v^{2}, \Lambda_{\mathrm{QCD}} / m_{\mathrm{b}}$, and even $\Lambda_{\mathrm{QCD}} / m_{\mathrm{c}}$ corrections besides this trivial phase-space factor. Hence these numbers should be regarded as an only very naive indication of the uncertainties due to these corrections.

We observe that, by stretching the poorly determined colour-singlet Wilson coefficient $2 C_{+}-C_{-}$, production of $\mathrm{J} / \psi$ and $\psi^{\prime}$ can be explained merely by the colour-singlet contribution, in contrast to $\chi_{\mathrm{c} J}$ production. This is in accord with the fact that there are two $\chi_{\mathrm{c} J}$ MEs in leading $v^{2}$ while only one $\psi$ ME survives for $v \rightarrow 0$. On the other hand, the values of the $\psi^{3} S_{1}$ colour-octet MEs are well compatible with the CLEO data for almost the whole range of $2 C_{+}-C_{-}$. In any case, the colour-octet ME combination $\mathcal{M}_{3.4}^{\psi}$ is too large, for example, by a factor of about 2 for the central value of $2 C_{+}-C_{-}$. This confirms the expectation raised above that it has been overestimated in fits to the Tevatron data.

An inspection of the $\chi_{\mathrm{c} 2}$ width reveals that the $\chi_{\mathrm{c} J}{ }^{3} S_{1}$ colour-octet ME is a factor of about 2 too large. The $\chi_{\mathrm{c} 1}$ width then requires the colour-singlet $\mathrm{ME}$ $\left\langle\mathcal{O}_{1}^{\chi c 1}\left({ }^{3} P_{1}\right)\right\rangle$ to be larger, by a factor of about 3 for the central value of $2 C_{+}-C_{-}$. This may well be consistent with the Tevatron data where there is a similar strong correlation between the two MEs.

While the leading-order $\chi_{\mathrm{c} J} \mathrm{ME}$ is rather well known, MEs of higher orders in $v^{2}$ have not yet been determined. However, relativistic corrections are likely to be important for $\chi_{\mathrm{c} J}$ production in fixed-target experiments and in $\mathrm{e}^{+} \mathrm{e}^{-}$collisions [21]. Corrections to $O\left(v^{2}\right)$ arise merely from operators with two extra spatial derivatives, but the same quantum numbers as the leading ones. Contributions that scale as $v^{4}$ relative to (20) give the correction

$$
\begin{aligned}
\Delta \mathrm{Br}\left[\mathrm{B} \rightarrow \chi_{\mathrm{c} J}+X\right]=\{ & 0.24\left\langle\mathcal{O}_{1}^{\chi_{\mathrm{c} J}}\left({ }^{3} S_{1}\right)\right\rangle+0.42\left\langle\mathcal{O}_{1}^{\chi_{\mathrm{c} J}}\left({ }^{1} S_{0}\right)\right\rangle \\
& \left.+21 \mathcal{M}_{3.4}^{\chi_{\mathrm{cJ} J}}+\frac{c_{D}}{m_{\mathrm{c}}^{4}}\left\langle\mathcal{O}_{8}^{\chi_{\mathrm{c} J}}\left({ }^{3} D_{J^{\prime}}\right)\right\rangle\right\} \mathrm{GeV}^{-3}
\end{aligned}
$$

where $c_{D}$ is an as yet uncalculated coefficient. Nonetheless, it is clear that B decays into $\chi_{\mathrm{c} J}$ states provide an upper limit on $\mathcal{M}_{3.4}^{\chi_{\mathrm{c} J}}$. Applying the scaling $v^{4} \sim 0.1$ to the two leading-order MEs, we find values for $\mathcal{M}_{3.4}^{\chi_{\mathrm{c} 1}}$ varying between $2 \times 10^{-3}$ and $1.5 \times 10^{-2}$. B-decay data certainly favour values at the lower end.

Let us finally mention that the $\chi_{\mathrm{c} 2}$-to- $\chi_{\mathrm{c} 1}$ ratio is a serious problem for the CEM, which predicts the ratio $5 / 3$. 


\section{Upsilon decay}

Inclusive charmonium production in $\Upsilon$ decays is another place to test the NRQCD

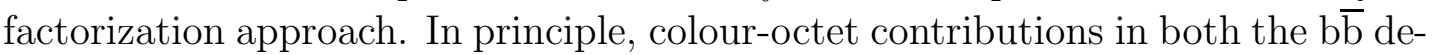
cay and the $c \overline{\mathrm{c}}$ production should be taken into account. However, relativistic corrections from the $\mathrm{b} \overline{\mathrm{b}}$ in a colour-octet state turn out to be small [22, in accordance with the naive expectation of a suppression by $\left(v_{\mathrm{b}} / v_{\mathrm{c}}\right)^{4} \sim 1 / 10$ relative to a contribution having the $c \bar{c}$ in a colour-octet state.

A full calculation of $\Upsilon$ decays into charmonia does not yet exist. The coloursinglet contribution to direct $\mathrm{J} / \psi$ production starts at $O\left(\alpha_{\mathrm{s}}^{6}\right)$ and consists of the tree diagrams $\mathrm{b} \overline{\mathrm{b}}_{1}\left({ }^{3} S_{1}\right) \rightarrow \mathrm{c}_{1}\left({ }^{3} S_{1}\right)+4 \mathrm{~g}$ and the loop diagrams $\mathrm{b} \overline{\mathrm{b}}_{1}\left({ }^{3} S_{1}\right) \rightarrow$ $\mathrm{c}_{1}\left({ }^{3} S_{1}\right)+2 \mathrm{~g}$ containing box diagrams. Only an estimate of the $O\left(\alpha_{\mathrm{s}}^{5}\right)$ coloursinglet contribution to indirect $\mathrm{J} / \psi$ production through $\chi_{\mathrm{c} J}$ decays exists, yielding about $1 / 20$ of the measured branching ratio [22, 23].

Colour-octet contributions to direct $\mathrm{J} / \psi$ production in $\Upsilon$ decays start at $O\left(\alpha_{\mathrm{s}}^{4}\right)$ and are hence enhanced by $1 / \alpha_{\mathrm{s}}^{2}$. Although calculations of the colour-singlet contributions are still lacking, a comparison with data can be used to set upper limits on certain colour-octet MEs. The largest colour-octet contribution found so far is $\mathrm{b}_{1}\left({ }^{3} S_{1}\right) \rightarrow$ ggg $^{\star}$ followed by $\mathrm{g}^{\star} \rightarrow \mathrm{c}_{8}\left({ }^{3} S_{1}\right)$ [22]. Another potentially large contribution is the loop process $\mathrm{b} \overline{\mathrm{b}}_{1}\left({ }^{3} S_{1}\right) \rightarrow \mathrm{c} \overline{\mathrm{c}}_{8}\left({ }^{1} S_{0},{ }^{3} P_{J}\right)+\mathrm{g}$ proceeding through virtual gluons. This process can be related ${ }^{[}$to the radiative decay $Q \bar{Q}_{1}\left({ }^{3} S_{1}\right) \rightarrow$ $\gamma+q \bar{q}_{1}\left({ }^{2 S+1} L_{J}\right)$ calculated some time ago [24]. We find

$$
\begin{aligned}
\operatorname{Br}[\Upsilon \rightarrow \mathrm{J} / \psi+X]= & \mathrm{BR}_{1}+R \frac{\pi \alpha_{\mathrm{s}}}{8\left(\pi^{2}-9\right) m_{\mathrm{c}}^{3}} \\
& \quad\left\{0.571\left\langle\mathcal{O}_{8}^{\mathrm{J} / \psi}\left({ }^{3} S_{1}\right)\right\rangle+0.90 \frac{\alpha_{\mathrm{s}}}{\pi} \mathcal{M}_{3.8}^{\mathrm{J} / \psi}\right\} \\
= & \mathrm{BR}_{1}+1.76 \times 10^{-2}\left\langle\mathcal{O}_{8}^{\mathrm{J} / \psi}\left({ }^{3} S_{1}\right)\right\rangle+0.247 \times 10^{-2} \mathcal{M}_{3.8}^{\mathrm{J} / \psi} \\
(11 \pm 4) \times 10^{-4}= & \mathrm{BR}_{1}+\{1.94+1.09\} \times 10^{-4}
\end{aligned}
$$

where $\mathrm{BR}_{1}$ denotes the missing part from unknown colour-singlet contributions and indirect $\mathrm{J} / \psi$ production. The second equation has been obtained with $R=$ $\operatorname{Br}[\Upsilon \rightarrow 3 \mathrm{~g}] \approx 0.83, \alpha_{\mathrm{s}}=0.278, m_{\mathrm{c}}=1.5$. The number on the LHS of the last line is the CLEO measurement [26] and the values of the RHS follow from insertion of the ME values in table 1. The latter are consistent with the measurement if, as expected, the colour-singlet part is small and the indirect contributions does not exceed, say, $50 \%$ of the total $\mathrm{J} / \psi$ yield. More definite conclusions can only be drawn once the missing pieces have been calculated and/or more experimental information is available, for example, on the $\mathrm{J} / \psi$ energy distribution or on the $\chi_{\mathrm{c} J}$ production rate.

\footnotetext{
${ }^{3}$ While finishing this paper we learnt that this process had just been taken up also in [25].
} 


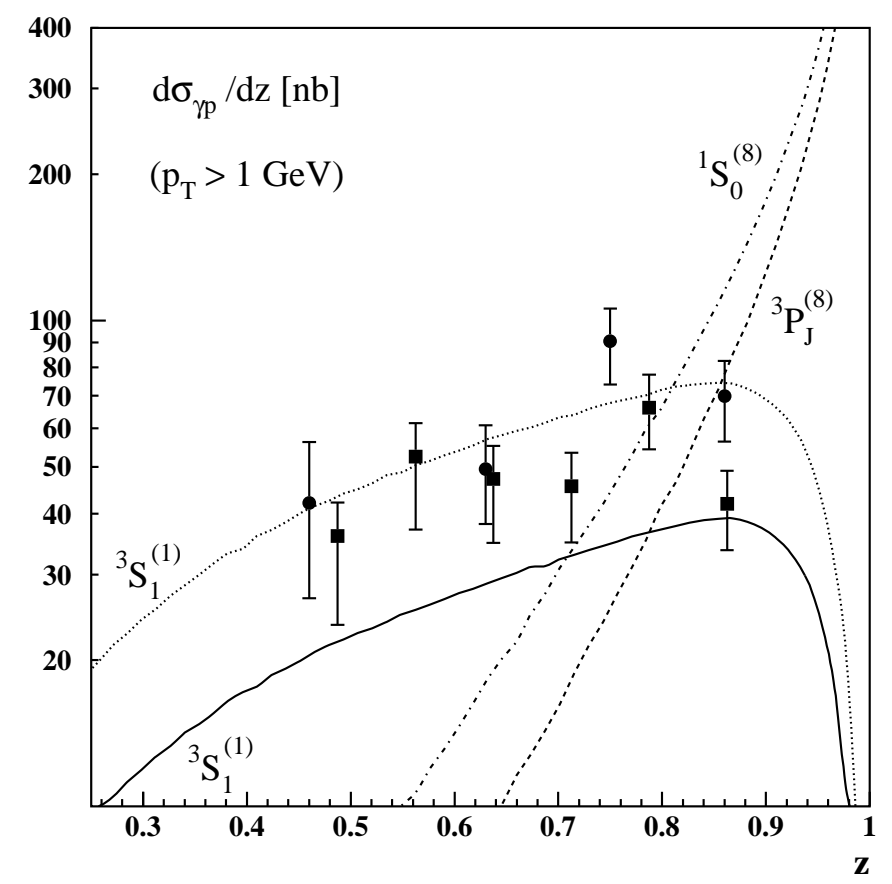

Figure 4: Photoproduction of $\mathrm{J} / \psi$ compared with HERA[28] data. Theoretical curves at $\sqrt{s}_{\gamma \mathrm{p}}=100 \mathrm{GeV}$ for $\left\langle\mathcal{O}_{1}^{\left.\mathrm{J} / \psi^{(3} S_{1}\right)}\right\rangle=1.16 \mathrm{GeV}^{3},\left\langle\mathcal{O}_{8}^{\mathrm{J} / \psi\left({ }^{1} S_{0}\right)}\right\rangle=0.03 \mathrm{GeV}^{3}$, $\left\langle\mathcal{O}_{8}^{\mathrm{J} / \psi}\left({ }^{3} P_{0}\right)\right\rangle=9 / 10^{3} \mathrm{GeV}^{3}, \alpha_{\mathrm{s}}=0.278$, and $m_{\mathrm{c}}=1.5 \mathrm{GeV}$, using the leadingorder GRV [30] parton distributions with $\mu=2 m_{\mathrm{c}}$. The colour-singlet contribution is shown also for $\alpha_{\mathrm{s}}=0.3, m_{\mathrm{c}}=1.4 \mathrm{GeV}$ (dotted line).

\section{Photoproduction}

Photoproduction of $\mathrm{J} / \psi$ has been claimed to be in conflict with NRQCD factorization [27]. In fact, the short-distance production of merely the leading coloursinglet state via

$$
\gamma+\mathrm{g} \rightarrow \mathrm{c} \overline{\mathrm{c}}_{1}\left({ }^{3} S_{1}\right)+\mathrm{g}
$$

yields a shape of the differential cross section $\mathrm{d} \sigma / \mathrm{d} z$, in good agreement with the HERA data [28] (Fig. (4), and also in agreement with low-energy data [29]. Here $z$ is the fraction of the photon energy carried by the $\mathrm{J} / \psi$ in the proton rest frame.

The absolute normalization suffers from uncertainties similar to the ones listed in section 2 for $\mathrm{J} / \psi$ production at the Tevatron: valuef of $m_{\mathrm{c}}$, parametrization of the parton-distribution functions, value of $\Lambda_{\mathrm{QCD}}$, choice of factorization and renormalization scales巳, and initial-state transverse-momentum effects, i.e. effects

\footnotetext{
${ }^{4}$ Note that the $m_{\mathrm{c}}$ dependence is partly compensated by corresponding changes in the ME; this has not been taken into account in Fig. 1 .

${ }^{5}$ The scale dependence has been reduced through the inclusion of next-to-leading order
} 
arising from the intrinsic transverse momentum $k_{\perp}$ of the gluon within the proton and initial-state gluon radiation. The latter effects mainly influence the $\mathrm{J} / \psi$ $p_{\perp}$-distribution at low $p_{\perp}$, but do affect the $z$ distribution as well, mostly its normalizations, less so its shape. This occurs in particular if a cut on $p_{\perp}$ is applied as low as $1 \mathrm{GeV}$, not much larger than $\left\langle k_{\perp}\right\rangle \sim 500 \mathrm{MeV}$. Recall that fixed-order calculations are reliable only at large $p_{\perp}$ or for $p_{\perp}$-integrated cross sections but do not adequately describe the shape at low $p_{\perp}$.

Relativistic corrections of order $v^{4}$ arise from

$$
\begin{aligned}
& \gamma+\mathrm{g} \rightarrow \mathrm{c} \overline{\mathrm{c}}_{8}[n]+\mathrm{g} \quad n={ }^{1} S_{0},{ }^{3} S_{1},{ }^{3} P_{J} \\
& \gamma+\mathrm{q} \rightarrow \mathrm{c} \overline{\mathrm{c}}_{8}[n]+\mathrm{q} \quad n={ }^{1} S_{0},{ }^{3} S_{1},{ }^{3} P_{J} \text {, }
\end{aligned}
$$

and from reactions involving the partonic content of the photon. Although such "resolved-photon processes" become more important as the c.m. energy increases, they still affect the small- $z$ region, only, say, below 0.3 at HERA energies. The quark-initiated reactions in (24) are small with respect to gluon-initiated ones at HERA energies. Moreover, $\gamma \mathrm{g} \rightarrow \mathrm{c} \overline{\mathrm{c}}_{8}\left({ }^{3} S_{1}\right)+\mathrm{g}$ is identical to (23), but down in magnitude by a factor of about 50. Most important are therefore the other two gluon-initiated reactions in (24). In fact, their contributions seem to be in clear conflict with the HERA data when using the MEs as given in table1, see Fig. 4 . This is not an artefact of the particular values of the $S$ - and $P$-wave MEs chosen in Fig. 4 , since both contributions are rather similar in shape. (To be precise, the value of $a$ in the ME combination (6) is $a=4.7 \pm 1.4$ in the range $0<z<1$ for $p_{\perp}>1 \mathrm{GeV}$.)

Three reasons make us believe that we do, in fact, not encounter a breakdown of NRQCD factorization, namely (i) the size of the ME, the treatment of (ii) the hard process and (iii) the hadronization. First, we have seen that all but the Tevatron estimates prefer smaller values of the $\mathrm{ME}$ combination $\mathcal{M}_{a \sim 5}^{\mathrm{J} / \psi}$. And we have given arguments why the value needed to explain the Tevatron data is likely to be smaller.

Second, (24) contains contributions that correspond to the evolution of the gluon (or quark) distribution functions. These terms arise from diagrams with $t$-channel gluon exchange and have to be removed via mass-factorization in calculations of fully $p_{\perp}$-integrated cross sections [32]. Cross sections integrated above some $p_{\perp}$-cut are reliably described by the fixed-order (in $\alpha_{\mathrm{s}}$ ) results based on (24) only if the cut is sufficiently large. If the $p_{\perp}$ cut is as low as, say, $1 \mathrm{GeV}$ then the average $p_{\perp}$ of the fixed-order calculation is considerably smaller than what would be obtained from a proper calculation including soft-gluon (initialstate) radiation. Since contributions from small $p_{\perp}$ lead, on average, to harder $z$ distributions and vice versa, we expect a flatter $z$ distribution after inclusion of initial-state radiation and intrinsic $k_{\perp}$.

perturbative corrections [31]. 
The third aspect concerns the sensitivity of the $z$ distribution to energy ranges in the $\mathrm{c}_{8}[n] \rightarrow \mathrm{J} / \psi$ transition of the order of the binding energy (and smaller). The physical reason is clear: the momentum carried away by light hadrons in the hadronization process makes it very unlikely that $z$ values close to 1 can be reached. Qualitatively, the hadronization causes a smearing of the $\mathrm{J} / \psi$ momentum over a region $\delta z \approx v^{2} \approx 0.25-0.30, \delta p_{\perp} \approx m v^{2} \approx 0.5 \mathrm{GeV}$. Technically, the NRQCD expansion parameter at $z$ close to one is $v^{2} /(1-\hat{z})$ rather than $v^{2}[\mathbb{8}$. The amount of smearing caused by this breakdown of the fixed-order (in $v^{2}$ ) calculation makes the latter unsuitable to predict the $z$ distribution. Predictivity can, however, be restored upon introduction of universal shape functions. Attempts computing these shape functions in the Coulomb limit $(m \rightarrow \infty)$ have started [33]. If supplemented by a sensible continuation into the higher-twist region, a $z$ distribution is obtained in agreement with the HERA data, provided the above-mentioned initial-state problem is dealt with as well. The required colour-octet MEs need not be much smaller than the naive estimates in table [1] [33].

\section{Conclusions}

The NRQCD factorization approach gives quarkonium production cross sections as a (finite) sum of short-distance coefficients times long-distance MEs. For the factorization to hold, the latter have to be process-independent. In this paper we have tested the universality of charmonium MEs by comparing their values as extracted from various reactions. Particular emphasis has been put to elucidate the uncertainties in such determinations. The results can be summarized as follows.

Up to now, the cleanest evidence for the need of short-distance production of colour-octet states in the formation of $1^{--}$mesons comes from the $\mathrm{J} / \psi$ and $\psi^{\prime} p_{\perp}$ distribution at the Tevatron. The numerical values of the corresponding colour-octet MEs are in line with the expectation from velocity scaling. Our investigation suggests that $\left\langle\mathcal{O}_{8}^{\psi}\left({ }^{3} S_{1}\right)\right\rangle$ is somewhat larger and $\mathcal{M}_{3.5}^{\psi}$ considerably smaller than currently extracted values (table @; $\psi$ denotes $\mathrm{J} / \psi$ or $\psi^{\prime}$ ).

In the case of $J^{++}$mesons: a colour-octet ME, $\left\langle\mathcal{O}_{8}^{\chi c 0}\left({ }^{3} S_{1}\right)\right\rangle$, enters already at leading order in $v^{2}$. Its numerical value as extracted from the Tevatron is highly correlated with the value of the colour-singlet $\mathrm{ME}\left\langle\mathcal{O}_{1}^{\chi_{1} 1}\left({ }^{3} P_{1}\right)\right\rangle$, whose value is less well known from potentail-model calculations than the colour-singlet $\psi$ ME. Indeed, B-meson decays into $\chi_{\mathrm{c} J}$ suggest a factor-of- 2 smaller $\left\langle\mathcal{O}_{8}^{\chi_{\mathrm{co}}}\left({ }^{3} S_{1}\right)\right\rangle$ value and a factor of 3 larger value for $\left\langle\mathcal{O}_{1}^{\chi_{1} 1}\left({ }^{3} P_{1}\right)\right\rangle$.

Although the full calculation of $\Upsilon$ decays into charmonia does not yet exist, the decay $\Upsilon \rightarrow \mathrm{J} / \psi+X$ does already provide upper limits on the $\mathrm{J} / \psi$ colour-

\footnotetext{
${ }^{6}$ Here $\hat{z}$ is defined analogously to $z$, i.e. $\hat{z}(z)$ is the photon-energy fraction carried by the c $\overline{\mathrm{c}}$ pair (the $\mathrm{J} / \psi$ ) in the proton rest frame.
} 
octet ME. If the colour-singlet contributions were really as small as suggested by their $\alpha_{\mathrm{s}}$ scaling, this would then not only prove that short-distance colouroctet production is at work but also result in a $\left\langle\mathcal{O}_{8}^{\mathrm{J} / \psi}\left({ }^{3} S_{1}\right)\right\rangle$ value larger than the current Tevatron estimate given in table 1 .

Similarly, $Z^{0}$ data at LEP are about a factor of 3 above the prediction based on colour-singlet production alone. Inclusion of colour-octet processes with $\left\langle\mathcal{O}_{8}^{\mathrm{J} / \psi}\left({ }^{3} S_{1}\right)\right\rangle$ as currently deduced from the Tevatron reconciles nicely the agreement with the LEP data.

Confronting theory and experiment of electron-positron annihilation into $\mathrm{J} / \psi X$ at two different low energies is in principle the best way to separate the two colour-octet MEs $\left\langle\mathcal{O}_{8}^{\mathrm{J} / \psi}\left({ }^{1} S_{0}\right)\right\rangle$ and $\left\langle\mathcal{O}_{8}^{\mathrm{J} / \psi}\left({ }^{3} P_{0}\right)\right\rangle$ that enter, in practically all other reactions, only in the linear combination (6) with almost constant $a$. However, current data suffer from statistics that are so low that they are compatible with pure colour-singlet production within 2 standard deviations. Neglecting the experimental errors, a value of $\mathcal{M}_{3.5}^{\mathrm{J} / \psi}$ is found somewhat smaller than the Tevatron one (table 11).

More accurate determinations of NRQCD MEs from $\mathrm{e}^{+} \mathrm{e}^{-}$annihilation and $\mathrm{Z}^{0}$ decays are mainly hindered by low statistics, and improvements can be expected with more data in the future. In contrast, constraints from $\Upsilon$ decays are currently limited by theory. The situation is similar for $\mathrm{B}$ decays into $\mathrm{J} / \psi$ and $\psi^{\prime}$ : The largest uncertainty arises from the Wilson coefficient $2 C_{+}-C_{-}$entering the effective weak Hamiltonian, which even in next-to-leading order is theoretically known only poorly. Pushing it to its maximum value, $\psi$ production is compatible with pure short-distance colour-singlet production. For most of the uncertainty range, however, the current Tevatron estimates for $\left\langle\mathcal{O}_{8}^{\psi}\left({ }^{3} S_{1}\right)\right\rangle$ are well compatible with data. On the other hand, $\mathcal{M}_{3.5}^{\psi}$ is once again found to be smaller than the Tevatron value in table 1, by about a factor of 2 .

Finally, photoproduction of $\mathrm{J} / \psi$ as measured at HERA does not pose a problem to NRQCD factorization, once colour-octet MEs compatible with all current data are being used, the $p_{\perp}$ broadening due to initial-state gluon radiation and intrinsic $k_{\perp}$ is taken into account, and the leading-order $v^{2}$ calculation is improved with the help of shape functions in order to extend its validity beyond a value of $z$ of about 0.7 .

\section{Acknowledgement}

It is my pleasure to thank G. Buchalla and M. Beneke for fruitful discussions. This work was supported in part by the EU Fourth Framework Programme "Training and Mobility of Researchers", Network "Quantum Chromodynamics and the Deep Structure of Elementary Particles", contract FMRX-CT98-0194 (DG 12MIHT). 


\section{References}

[1] G.T. Bodwin, E. Braaten and G.P. Lepage, Phys. Rev. D51 (1995) 1125.

[2] E. Braaten and T.C. Yuan, Phys. Rev. Lett. 71 (1993) 1673.

[3] M. Cacciari and M. Greco, Phys. Rev. Lett. 73 (1994) 1586;

E. Braaten, M. Doncheski, S. Fleming and M. Mangano, Phys. Lett. B333 (1994) 548;

D.P. Roy and K. Sridhar, Phys. Lett. B339 (1994) 141;

E. Braaten and S. Fleming, Phys. Rev. Lett. 74 (1995) 3327;

M. Cacciari, M. Greco, M. Mangano and A. Petrelli, Phys. Lett. B356 (1995) 560 ;

P. Cho and A.K. Leibovich, Phys. Rev. D53 (1996) 150, ibid. 6203;

M. Beneke and M. Krämer, Phys. Rev. D55 (1997) 5269.

[4] For reviews see E. Braaten, S. Fleming and T.C. Yuan, Ann. Rev. Nucl. Part. Sci. 46 (1996) 197;

M. Beneke, hep-ph/9703429, in 24th Annual SLAC Summer Institute on Particle Physics: The Strong Interaction, From Hadrons to Protons (SSI 96), Stanford, USA, 1996, p. 549;

M. Cacciari, hep-ph/9706374, talk given at 32nd Rencontres de Moriond, Les Arcs, France, 1997.

[5] F. Abe et al., Phys. Rev. Lett. 79 (1997) 572, ibid. 578;

A. Sansoni, for the CDF collab., Nucl. Phys. A610 (1996) 373c.

[6] B. Cano-Coloma and M.A. Sanchis-Lozano, Nucl. Phys. B508 (1997) 753;

P. Ernström, L. Lönnblad and M. Vanttinen, Z. Phys. C76 (1997) 515;

B.A. Kniehl and G. Kramer, hep-ph/9803256.

[7] G.A. Schuler, CERN-TH/7170-94, hep-ph/9403387.

[8] M. Beneke, I.Z. Rothstein and M.B. Wise, Phys. Lett. B408 (1997) 373.

[9] E.J. Eichten and C. Quigg, Phys. Rev. D52 (1995) 1726.

[10] G. A. Schuler, Int. J. Mod. Phys. A12 (1997) 3951.

[11] DELPHI collab., P. Abreu et al., Phys. Lett. B341 (1994) 109;

M. Wadhwa for the L3 collab., talk given at QCD97, Montpellier, 1997;

ALEPH collab., contribution 624 to the 1997 EPS-HEP conference, Jerusalem, 1997;

OPAL collab., Phys. Lett. B384 (1996) 343. 
[12] DELPHI collab., P. Abreu et al., Z. Phys. C69 (1996) 575;

ALEPH collab., contribution PA05-066 to the EPS-HEP conference, Warsaw, 1996;

L3 collab., M. Acciarri et al., CERN-PPE/97-78, July 1997;

OPAL collab., Phys. Lett. B370 (1996) 185.

[13] For a review see, for example, G.A. Schuler, Z. Phys. C71 (1996) 317.

[14] G.A. Schuler and R. Vogt, Phys. Lett. B387 (1996) 181.

[15] J.H. Kühn and H. Schneider, Z. Phys. C11 (1981) 263;

W.-Y. Keung, Phys. Rev. D23 (1981) 2072;

L. Clavelli, Phys. Rev. D26 (1982) 1610;

V. Barger, K. Cheung and W.-Y. Keung, Phys. Rev. D41 (1990) 1541;

E. Braaten, K. Cheung and T.C. Yuan, Phys. Rev. D48 (1993) 4230;

V.M. Driesen, J.H. Kühn and E. Mirkes, Phys. Rev. D49 (1994) 3197;

P. Cho and A.K. Leibovich, Phys. Rev. D54 (1996) 6690;

P. Cho, Phys. Lett. B368 (1996) 171;

K. Cheung, W.-Y. Keung and T.C. Yuan, Phys. Rev. D76 (1996) 877;

S. Baek, P. Ko, J. Lee and H.S. Song, Phys. Lett. B389 (1996) 609, Phys. Rev. D55 (1997) 6839.

[16] F. Yuan, C.-F. Qiao, K.-T. Chao, Phys. Rev. D56 (1997) 321, ibid. 1663.

[17] PLUTO collab., J. Burmester et al., Phys. Lett. B68 (1977) 283;

R.A. Poling, for the CLEO collab., in Proc. Joint Int. Lepton-Photon Symposium 86 Europhysics Conf. on High-Energy Physics, Geneva, 1991, eds. S. Hegarty et al. (World Scientific, Singapore, 1992), p. 546.

[18] T. Mannel and G.A. Schuler, Z. Phys. C67 (1995) 159.

[19] CLEO collab., R. Balest et al., Phys. Rev. D52 (1995) 2661.

[20] P. Ko, J. Lee and H.S. Song, Phys. Rev. D53 (1996) 1409;

S. Fleming et al., Phys. Rev. D55 (1997) 4098.

[21] G.A. Schuler and M. Vänttinen, CERN-TH-97-261, October 1997, hep$\mathrm{ph} / 9710227$.

[22] K. Cheung, W.-Y. Keung and T.C. Yuan, Phys. Rev. D54 (1996) 929.

[23] H. Trottier, Phys. Lett. B320 (1994) 145.

[24] J.G. Körner, J.H. Kühn, M. Krammer and H. Schneider, Nucl. Phys. B299 (1983) 115.

[25] M. Napsuciale, hep-ph/9710488. 
[26] CLEO collab., R. Fulton et al., Phys. Lett. B224 (1989) 445.

[27] M. Cacciari and M. Krämer, Phys. Rev. Lett. 76 (1996) 4128;

P. Ko, J. Lee and H.S. Song, Phys. Rev. D54 (1996) 4312.

[28] H1 collab., S. Aid et al., Nucl. Phys. B472 (1996) 3;

ZEUS collab., J. Breitweg et al., Z. Phys. C76 (1997) 599.

[29] H. Jung, G.A. Schuler and J. Terron, Int. J. Mod. Phys. A7 (1992) 7955.

[30] M. Glück, E. Reya and A. Vogt, Z. Phys. C53 (1992) 127.

[31] M. Krämer, J. Zunft, J. Steegborn and P.M. Zerwas, Phys. Lett. B348 (1995) 657; M. Krämer, Nucl. Phys. B459 (1996) 3.

[32] F. Maltoni, M. Mangano and A. Petrelli, hep-ph/9708349; A. Petrelli, M. Cacciari, M. Greco, F. Maltoni and M. Mangano, Nucl. Phys. B514 (1998) 245.

[33] M. Beneke, G.A. Schuler and S. Wolf, CERN preprint in preparation. 